\title{
Herramientas Web 2.0 y su relación con la convivencia escolar: valoraciones del educador social
}

\author{
Web 2.0 tools and their relationship with school coexistence: social \\ educator ratings
}

\author{
Begoña Esther Sampedro Requena \\ Universidad de Córdoba, España (bsampedro@uco.es)
}

Recibido el 16 de noviembre de 2016; revisado el 23 de noviembre de 2016; aceptado el 23 de noviembre de 2016; publicado el 2 de diciembre de 2016

\section{RESUMEN:}

La educación para la convivencia y la resolución de conflictos se han convertido para las instituciones escolares y para los equipos de orientación educativa en eje clave de actuación; la diversidad de contextos en los que nos desarrollamos y las actuales formas de interacción, propiciadas por las tecnologías, han generado nuevas conductas y comportamientos para convivir que deben ser abordados por diversos agentes y diferentes métodos. El objetivo de esta investigación es conocer la valoración que expresa el alumnado de Grado de Educador Social de una batería de once herramientas Web 2.0 para la intervención educativa en el ámbito de la educación para la convivencia y resolución de conflictos, mediante una metodología de carácter cuantitativa basada en un diseño ex post facto. Los resultados muestran que las redes sociales y los buscadores de vídeo son los recursos tecnológicos mejor valorados, para la aplicación en este ámbito de intervención, por los sujetos participantes; por otro lado, la estimación se encuentra condicionada por diferentes aspectos como el curso en el que se encuentran adscritos los estudiantes, la frecuencia temporal de acceso a internet, el tipo de conexión o el dispositivo que emplean.
PALABRAS CLAVE: TECNOLOGÍAS WEB 2.0, RESOLUCIÓN DE CONFLICTOS, EDUCACIÓN COMUNITARIA.

\begin{abstract}
:
Education for coexistence and conflict resolution have become for school institutions and for educational counseling teams in key action axis; the diversity of contexts in which we develop and the current forms of interaction, propitiated by the technologies, have generated new actions and behaviors to coexist that must be approached by different agents and different methods. The objective of this research is to know the assessment expressed by the students of Social Educator Degree of a battery of eleven Web 2.0 tools for educational intervention in the field of education for coexistence and conflict resolution, using a quantitative methodology Based on an ex post facto design. The results show that social networks and vídeo search engines are the most rated technological resources for the application in this field of intervention by the participating subjects. Moreover, the estimation is conditioned by different aspects such as the degree year are assigned, the temporal frequency of Internet access, the type of connection or the device they use.
\end{abstract}

KEYWORDS: WEB 2.0 TECHNOLOGIES,
CONFLICTO RESOLUTION, COMMUNITY
EDUCATION.




\section{INTRODUCCIÓN}

Si entendemos la escuela o los centros escolares como un espacio donde se entablan diversas relaciones, en el que se interactúa y convive, parece lógico pensar que es necesario abordar aspectos como la educación para la convivencia y la resolución de conflictos.

Como señala Bisquerra (2008) la educación para la convivencia desarrolla diversas destrezas, valores y actitudes que fomentan la competencia ciudadana, entre los que destacan "respecto; escuchar; diálogo; empatía; asertividad; negociación; regulación emocional (sobre todo en situaciones de conflicto); mantener la calma; responsabilidad; solidaridad; criterios éticos, etc." (p. 91).

Los centros escolares, como comunidades educativas en las que se desarrollan actos educativos que necesitan la comunicación, el diálogo y la interacción, necesitan la inclusión de diversos agentes de diferentes campos profesionales, entre los que se encuentra el educador social.

Estos profesionales no pueden mantenerse al margen de las tendencias actuales, tanto educativas como sociales, lo cual implica el empleo de los medios tecnológicos en los actos educativos, que condicionan el comportamiento y las acciones, por tanto la convivencia y las relaciones.

Estudios internacionales como el de EscorciaOyola y Jaimes de Triviño (2015) ponen de manifiesto la importancia de emplear las herramientas Web 2.0 en proyectos educativos, señalando, en concreto, los beneficios para la convivencia escolar y la resolución de conflictos.

Por otro lado, las investigaciones llevadas a cabo por Fullana, Pallisera y Planas (2011); González, Olmos y Serrate (2015); Rodríguez Izquierdo (2011), reflejan que el educador social se encuentra en España totalmente integrado en los centros educativos, formando parte de los proyectos y acciones educativas encaminadas a la integración activa de los sujetos a la vida escolar, desarrollando en ellos hábitos y actitudes sociales y personales.

En consecuencia, es necesario abordar qué estimación poseen los futuros educadores sociales en relación a las herramientas Web 2.0 para su aplicación en los entornos escolares en la búsqueda del desarrollo de la convivencia y la resolución de conflictos, como aspectos clave de la vida escolar.

\section{METODOLOGÍA Y DISEÑO}

Esta investigación plantea, como objetivo general, determinar el grado de valoración estimado por los estudiantes del Grado de Educador Social de la Universidad Pablo Olavide (Sevilla) de una batería de once herramientas Web 2.0 para el ámbito de intervención en la educación para la convivencia y resolución de conflictos.

La orientación de la misma se basa en un paradigma positivista, caracterizado por describir, relacionar, explicar y predecir variables (Meza, 2002), mediante un enfoque empírico - analítico y/o cuantitativo, donde se engloba el diseño ex post facto (Sabariego, 2012).

El instrumento diseñado ad hoc para la recogida de los datos, posee los elementos básicos de rigor científico, dado que tiene una alta confiabilidad como lo demuestra el valor de la prueba aplicada para el enfoque de consistencia interna (Morales, 2007), procedimiento de Alfa de Cronbach 0.920; por otro lado, buscando la fortaleza en el carácter unidimensional de la prueba (índice de fiabilidad) se ha realizado un análisis de la capacidad de discriminación de los elementos a través de la prueba estadística $\mathrm{t}$ de Student (para muestras independientes) entre las medias de los grupos establecidos, revelando un alto poder de discriminación en todos los ítems (valores de significatividad $\mathrm{p}=0.000$ ), lo cual implica que los elementos recogidos en la prueba cumplen con el objetivo planteado.

Además, al cuestionario elaborado se le aplicó la prueba de juicio de expertos mediante Panel Internacional de Investigación en Tecnología Educativa (PI2TE) de la asociación EDUTEC (http://gte2.uib.es/panel), los cuales reflejaron la óptima claridad, pertinencia y secuenciación del instrumento.

Asimismo, en referencia a la validez de constructo, el análisis factorial exploratorio aplicado, mediante la extracción de análisis de componentes principales, con un método de rotación de normalización varimax con Kaiser, revela que el índice Kaiser - Meyer - Olkin (KMO) es de 0.876 y el test de esfericidad de Barllett (con $\mathrm{p}=0.000)$, y una varianza total explicada de $68.5 \%$; hecho que implica una aceptable validez (Hernández, Fernández y Baptista, 2014).

El muestreo aplicado para este estudio se basa en un tipo causal (Sabariego 2012), característico en investigaciones del ámbito de las ciencias sociales y educativas, basado en la facilidad de acceso a los individuos objeto de estudio. La muestra está formada por estudiantes del Grado de Educación Social de la Universidad Pablo de Olavide (Sevilla), donde el 27,7\% son de primer curso; el 26,6\% de segundo; el $24,4 \%$ de tercero y el $21,3 \%$ de cuarto. 
En relación al sexo, el $82,9 \%$ son mujeres y el 17,1\% hombres. La distribución de todos ellos, relativa al dispositivo que emplean para su conexión a Internet, es: $25,1 \%$ el móvil, $68,8 \%$ el PC y el $6 \%$ la tableta. Finalmente, considerando la frecuencia semanal de conexión, el $13,6 \%$ la realiza dos o tres días, el $33,2 \%$ cuatro o cinco y el 53,3\% todos los días de la semana; igualmente, al considerar en esta ocasión el acceso a la red por horas al día, el 37,2\% lo hacen una o dos horas, el $41,2 \%$ entre tres o cuatro y el $21,6 \%$ más de cinco.

\section{RESULTADOS}

Los estudiantes del Grado de Educador Social de la Universidad Pablo de Olavide (Sevilla) valoran con indiferencia los buscadores de texto (promedio $=3,10$ ), los editores de vídeo (promedio $=3,39$ ), los entornos de aprendizaje virtuales y los editores de imágenes (ambos promedios $=3,40$ ), los buscadores de presentaciones (promedio $=3,46$ ), los buscadores de imágenes y los foros (ambos promedios=3,48), los blogs (promedio $=3,49)$ para la intervención en el ámbito de la educación para la convivencia y resolución de conflictos.

Asimismo señalan de forma más positiva las redes sociales y educativas (promedio $=3,63$ ) y los buscadores de vídeo (promedio $=3,58$ ); por otra parte, la wiki (promedio $=2,45)$ la valoran negativamente para intervenir en este ámbito de trabajo.

\subsection{Valoración herramientas Web 2.0 en función del sexo}

En lo que se refiere al el sexo podemos señalar que no es una variable que produzca diferencia en la dimensión de la valoración de algunas herramientas Web 2.0 para la educación para la convivencia y resolución de conflictos, los mismos se extraen de la aplicación de una prueba T de Student (n.s.=0.05).

\subsection{Valoración herramientas Web 2.0 en función del curso}

El curso en el que se encuentra el alumnado también es susceptible de comparativa; por esta razón se ha efectuado una Anova (n.s.=0.05) obteniendo los siguientes valores representativos que se muestran en la tabla 2.

Los estudiantes pertenecientes al primer curso indican una valoración cercana a positiva de los entornos de aprendizaje virtuales para el ámbito de la educación para la convivencia y resolución de conflictos con respecto a los de tercero $\mathrm{t}(185)=3.943$ y $\mathrm{p}=0.001 ; \mathrm{y}$ a los de cuarto $\mathrm{t}(185)=3.036$ y $\mathrm{p}$ $=0.016$. Igualmente la comparativa con respecto a la valoración de esta herramienta Web 2.0 entre el curso de segundo y tercero arroja resultados significativos, con mejor apreciación de los niveles inferiores $\mathrm{t}(185)=3.149$ y $\mathrm{p}=0.011$.

El alumnado que cursa primero valora positivamente los blogs para este ámbito de intervención frente a los de tercero $\mathrm{t}(185)=4.856$ y $\mathrm{p}=0.000 ; \mathrm{y}$ a los de cuarto $\mathrm{t}(185)=3.714 \mathrm{y} \mathrm{p}=0.002$. Equivalentemente, el alumnado de segundo sí presenta resultados relevantes en la comparación con los de tercero, valorando más positivamente esta herramienta Web 2.0 en la intervención de la educación para la convivencia y resolución de conflictos $\mathrm{t}(185)=3.490$ y $\mathrm{p}=0.004$.

Los estudiantes pertenecientes al primer curso realizan una valoración cercana a positiva de las redes sociales y educativas en relación a los de tercero $\mathrm{t}(185)=3.443$ y $\mathrm{p}=0.004 ; \mathrm{y}$ a los de cuarto $\mathrm{t}(185)=2.870 \mathrm{p}=0.027$; asimismo, el alumnado de segundo valora positivamente esta herramienta Web 2.0 con respecto a los de tercero $\mathrm{t}(185)=3.846$ y $\mathrm{p}=0.001 \mathrm{y}$, con los de cuarto $\mathrm{t}(185)=3.263$ y $\mathrm{p}=0.008$.

La comparativa considerando la valoración casi positiva de los editores de vídeo para la intervención en la educación para la convivencia y resolución de conflictos del alumnado de primero frente al de tercero, es más favorable en los cursos inferiores, $\mathrm{t}(185)=4.495$ y $\mathrm{p}=0.000$; igualmente con los de cuarto $\mathrm{t}(185)=3.132$ y $\mathrm{p}=0.012$. Mientras que el alumnado adscrito a segundo valora indiferente esta herramienta Web 2.0 para este ámbito frente a los de tercero, cuya valoración es negativa $\mathrm{t}(185)=3.715 \mathrm{y}$ $\mathrm{p}=0.002$.

Los editores de imágenes son mejor valorados para la intervención en este ámbito por los estudiantes adscritos a primero que por los de tercero $\mathrm{t}(185)=3.678$ y $\mathrm{p}=0.002$; asimismo el alumnado de segundo valora el ámbito mejor que tercero $\mathrm{t}(185)=3.588$ y $\mathrm{p}=0.002$. En cambio no podemos afirmar los mismos resultados para el curso de cuarto dado que los valores del análisis de la tasa de error no son indicativos.

Los estudiantes pertenecientes al primer curso realizan una valoración cercana a positiva de los buscadores de imágenes en el ámbito de intervención de la educación para la convivencia y resolución de conflictos con respecto a los de tercero $\mathrm{t}(185)=3.646$ y $\mathrm{p}=0.002 ;$ y a los de cuarto $\mathrm{t}(185)=3.026$ y $\mathrm{p}=0.017$; asimismo el alumnado de segundo valora casi positivamente esta herramienta 
Web 2.0 con respecto a los de tercero $t(185)=3.932$ y $\mathrm{p}=0.001 \mathrm{y}$, con los de cuarto $\mathrm{t}(185)=3.301 \mathrm{y}$ $\mathrm{p}=0.007$.

Asimismo, la herramienta Web 2.0 buscadores de vídeo se valora más positivamente por la muestra de primero que por la de tercero $t(185)=2.695$ y $\mathrm{p}=0.046$; por el contario no se evidencian datos indicativos con la comparación de varianzas del resto de los cursos.

El alumnado que cursa primero valora positivamente los buscadores de texto para este ámbito frente a los de tercero $\mathrm{t}(185)=4.700 \mathrm{y}$ $\mathrm{p}=0.001 \mathrm{y}$, a los de cuarto $\mathrm{t}(185)=3.553$ y $\mathrm{p}=0.003$. Equivalentemente, el alumnado de segundo si presenta resultados relevantes en la comparación con los de tercero, valorando más positivamente esta herramienta Web 2.0 en la intervención de la educación para la convivencia y resolución de conflictos $\mathrm{t}(185)=4.392$ y $\mathrm{p}=0.001$; asimismo con los de cuarto $\mathrm{t}(185)=3.269$ y $\mathrm{p}=0.008$.

Los estudiantes pertenecientes al primer curso indica una valoración cercana a positiva de los buscadores de presentaciones respecto a los de tercero $\mathrm{t}(185)=3.120$ y $\mathrm{p}=0.012$. Igualmente la comparativa entre el curso de segundo y tercero arroja resultados significativos $\mathrm{t}(185)=4.581$ y $\mathrm{p}=0.000$, y segundo frente a cuarto $\mathrm{t}(185)=3.294 \mathrm{y}$ $\mathrm{p}=0.007$, con mejor apreciación de los niveles inferiores.

La comparativa considerando la valoración casi positiva de la wiki para la intervención en la educación para la convivencia y resolución de conflictos del alumnado de primero frente al de tercero, es más favorable en los cursos inferiores, $\mathrm{t}(185)=4.795 \mathrm{y} \mathrm{p}=0.001$; igualmente sucede con los de cuarto $t(185)=3.054$ y $p=0.016$. Mientras que el alumnado adscrito a segundo valora casi positivamente esta herramienta Web 2.0 frente a los de tercero, cuya valoración es negativa $\mathrm{t}(185)=4.094$ y $\mathrm{p}=0.000$.

Tabla 1. Anova de la valoración del ámbito de intervención de la educación para la convivencia y resolución de conflictos de once herramientas Web 2.0 en función de los cursos a los que se adscribe la muestra.

\begin{tabular}{|c|c|c|c|c|c|}
\hline \multirow{5}{*}{ Entornos de aprendizaje virtuales } & Curso & $\mathrm{N}$ & Media & $\mathrm{s}$ & F y p de los grupos favorables \\
\hline & $1^{\mathrm{o}}$ & 52 & 3.80 & 0.786 & \multirow{4}{*}{$\begin{array}{c}\mathrm{F}=6.948 \text { y } \mathrm{p}=0.001 \\
1^{\mathrm{o}}>3^{\mathrm{o}} ; 1^{\mathrm{o}}>4^{\mathrm{o}} \\
2^{\mathrm{o}}>3^{\mathrm{o}}\end{array}$} \\
\hline & $2^{\circ}$ & 50 & 3.63 & 0.991 & \\
\hline & $3^{\circ}$ & 46 & 2.96 & 1.385 & \\
\hline & $4^{\circ}$ & 40 & 3.12 & 1.109 & \\
\hline \multirow{4}{*}{ Blogs } & $1^{\mathrm{o}}$ & 52 & 3.98 & 0.714 & \multirow{4}{*}{$\begin{array}{c}\mathrm{F}=9.871 \mathrm{y} \mathrm{p}=0.000 \\
1^{\mathrm{o}}>3^{\mathrm{o}} ; 1^{\mathrm{o}}>4^{\mathrm{o}} \\
2^{\mathrm{o}}>3^{\mathrm{o}}\end{array}$} \\
\hline & $2^{\circ}$ & 50 & 3.71 & 0.893 & \\
\hline & $3^{\circ}$ & 46 & 3.00 & 1.296 & \\
\hline & $4^{\circ}$ & 40 & 3.19 & 1.174 & \\
\hline \multirow{4}{*}{ Redes sociales y educativas } & $1^{\mathrm{o}}$ & 52 & 3.93 & 0.887 & \multirow{4}{*}{$\begin{array}{c}\mathrm{F}=7.707 \text { y } \mathrm{p}=0.000 \\
1^{\mathrm{o}}>3^{\mathrm{o}} ; 1^{\mathrm{o}}>4^{\mathrm{o}} \\
2^{\mathrm{o}}>3^{\mathrm{o}} ; 2^{\mathrm{o}}>4^{\mathrm{o}}\end{array}$} \\
\hline & $2^{\circ}$ & 50 & 4.02 & 0.804 & \\
\hline & $3^{\circ}$ & 46 & 3.20 & 1.497 & \\
\hline & $4^{\circ}$ & 40 & 3.29 & 1.019 & \\
\hline
\end{tabular}

El foro como herramienta Web 2.0 es mejor valorada para la intervención en este ámbito por los estudiantes adscritos a primero que por los de tercero $\mathrm{t}(185)=3.359$ y $\mathrm{p}=0.006$. Asimismo el alumnado de segundo valora mejor que tercero $\mathrm{t}(185)=3.936$ y $\mathrm{p}=0.001$ y, que el de cuarto $\mathrm{t}(185)=3.125$ y $\mathrm{p}=0.012$. 


\begin{tabular}{|c|c|c|c|c|c|}
\hline \multirow{4}{*}{ Editores de vídeo } & $1^{\circ}$ & 52 & 3.83 & 0.795 & \multirow{4}{*}{$\begin{array}{c}\mathrm{F}=8.700 \text { y } \mathrm{p}=0.000 \\
1^{\circ}>3^{\mathrm{o}} ; 1^{\mathrm{o}}>4^{\mathrm{o}} \\
2^{\mathrm{o}}>3^{\mathrm{o}}\end{array}$} \\
\hline & $2^{\circ}$ & 50 & 3.67 & 0.964 & \\
\hline & $3^{\circ}$ & 46 & 2.86 & 1.357 & \\
\hline & $4^{\circ}$ & 40 & 3.12 & 1.273 & \\
\hline \multirow{4}{*}{ Editores de imágenes } & $1^{\mathrm{o}}$ & 52 & 3.70 & 0.792 & \multirow{4}{*}{$\begin{array}{c}\mathrm{F}=6.731 \mathrm{y} \mathrm{p}=0.000 \\
1^{\circ}>3^{\circ} \\
2^{\circ}>3^{\circ}\end{array}$} \\
\hline & $2^{o}$ & 50 & 3.69 & 0.919 & \\
\hline & $3^{\circ}$ & 46 & 2.96 & 1.311 & \\
\hline & $4^{\mathrm{o}}$ & 40 & 3.17 & 1.057 & \\
\hline \multirow{4}{*}{ Buscadores de imágenes } & $1^{\mathrm{o}}$ & 52 & 3.81 & 0.779 & \multirow{4}{*}{$\begin{array}{c}\mathrm{F}=8.257 \text { y } \mathrm{p}=0.000 \\
1^{\mathrm{o}}>3^{\mathrm{o}} ; 1^{\mathrm{o}}>4^{\mathrm{o}} \\
2^{\mathrm{o}}>3^{\mathrm{o}} ; 2^{\mathrm{o}}>4^{\mathrm{o}}\end{array}$} \\
\hline & $2^{\circ}$ & 50 & 3.88 & 0.900 & \\
\hline & $3^{\circ}$ & 46 & 3.02 & 1.476 & \\
\hline & $4^{\mathrm{o}}$ & 40 & 3.12 & 1.214 & \\
\hline \multirow{4}{*}{ Buscadores de vídeo } & $1^{\mathrm{o}}$ & 52 & 4.44 & 5.538 & \multirow{4}{*}{$\begin{array}{c}\mathrm{F}=2.762 \text { y } \mathrm{p}=0.043 \\
1^{\circ}>3^{\circ}\end{array}$} \\
\hline & $2^{o}$ & 50 & 3.75 & 0.905 & \\
\hline & $3^{\circ}$ & 46 & 2.84 & 1.347 & \\
\hline & $4^{\mathrm{o}}$ & 40 & 3.17 & 1.080 & \\
\hline \multirow{4}{*}{ Buscadores de texto } & $1^{\mathrm{o}}$ & 52 & 4.00 & 0.752 & \multirow{4}{*}{$\begin{array}{c}\mathrm{F}=11.067 \text { y } \mathrm{p}=0.000 \\
1^{\mathrm{o}}>3^{\mathrm{o}} ; 1^{\mathrm{o}}>4^{\mathrm{o}} \\
2^{\mathrm{o}}>3^{\mathrm{o}} ; 2^{\mathrm{o}}>4^{\mathrm{o}}\end{array}$} \\
\hline & $2^{\circ}$ & 50 & 3.94 & 0.802 & \\
\hline & $3^{\circ}$ & 46 & 2.98 & 1.421 & \\
\hline & $4^{\circ}$ & 40 & 3.19 & 1.366 & \\
\hline \multirow{4}{*}{ Buscadores de presentaciones } & $1^{\mathrm{o}}$ & 52 & 3.63 & 0.760 & \multirow{4}{*}{$\begin{array}{c}\mathrm{F}=8.193 \text { y } \mathrm{p}=0.000 \\
1^{\mathrm{o}}>3^{\mathrm{o}} \\
2^{\mathrm{o}}>3^{\mathrm{o}} ; 2^{\mathrm{o}}>4^{\mathrm{o}}\end{array}$} \\
\hline & $2^{\circ}$ & 50 & 3.94 & 0.873 & \\
\hline & $3^{\circ}$ & 46 & 2.98 & 1.435 & \\
\hline & $4^{\mathrm{o}}$ & 40 & 3.21 & 1.094 & \\
\hline \multirow{4}{*}{ Wiki } & $1^{\mathrm{o}}$ & 52 & 3.89 & 0.793 & \multirow{4}{*}{$\begin{array}{c}\mathrm{F}=9.759 \text { y } \mathrm{p}=0.000 \\
1^{\circ}>3^{\mathrm{o}} ; 1^{\circ}>4^{\mathrm{o}} \\
2^{\mathrm{o}}>3^{\mathrm{o}}\end{array}$} \\
\hline & $2^{o}$ & 50 & 3.75 & 0.968 & \\
\hline & $3^{\circ}$ & 46 & 2.88 & 1.321 & \\
\hline & $4^{\circ}$ & 40 & 3.21 & 1.180 & \\
\hline \multirow{4}{*}{ Foros } & $1^{\mathrm{o}}$ & 52 & 3.74 & 0,805 & \multirow{4}{*}{$\begin{array}{c}\mathrm{F}=7.356 \text { y } \mathrm{p}=0.000 \\
1^{\circ}>3^{\mathrm{o}} \\
2^{\mathrm{o}}>3^{\mathrm{o}} ; 2^{\mathrm{o}}>4^{\mathrm{o}}\end{array}$} \\
\hline & $2^{\mathrm{o}}$ & 50 & 3.87 & 0,841 & \\
\hline & $3^{\circ}$ & 46 & 3.06 & 1,348 & \\
\hline & $4^{o}$ & 40 & 3.19 & 1,110 & \\
\hline
\end{tabular}

\subsection{Valoración herramientas Web 2.0 en función del dispositivo que emplean frecuentemente}

Contemplando en esta ocasión el dispositivo que emplea habitualmente la muestra participante, hemos efectuado una Anova (n.s.=0.05), cuyos resultados reflejan que:

El alumnado que emplea el móvil como dispositivo de acceso a Internet valora casi positivamente los entornos de aprendizaje virtuales $\mathrm{F}(2,186)=3.916$ y $\mathrm{p}=0.021$. En relación con los del Pc $\mathrm{t}(186)=2.565$ y $\mathrm{p}=0.034$, mientras que la comparación entre el móvil y la tableta no arrojan resultados notables en las pruebas post hoc aplicadas.

La muestra participante que dispone de un móvil para la conexión a Internet valora más positivamente los blogs $\mathrm{F}(2,186)=5.584$ y $\mathrm{p}=0.004$, para la intervención en el ámbito de la educación para la convivencia y resolución de conflictos en comparación con los del $\mathrm{Pc}$ en todas sus modalidades ordenador de mesa, portátil $\mathrm{t}(186)=3.140$ y $\mathrm{p}=0.006$; por el contrario no podemos afirmar los mismos resultados para el dispositivo tableta dado que los valores del análisis de la tasa de error no son indicativos.

La valoración casi positiva de los editores de vídeo $\mathrm{F}(2,186)=3.650$ y $\mathrm{p}=0.028$, se confiere en los estudiantes que emplean asiduamente el móvil como dispositivo de acceso a Internet respecto a los del PC $\mathrm{t}(186)=2.680 \mathrm{y} \mathrm{p}=0.024$, las restantes comparaciones no provocan significación.

Los estudiantes que emplean el móvil como dispositivo de acceso a Internet valoran casi positivamente los buscadores de texto $\mathrm{F}(2,186)=3.643$ y $\mathrm{p}=0.028$ para la intervención en el ámbito de la educación para la convivencia y resolución de conflictos frente a los del PC $\mathrm{t}(186)=2.469$ y $\mathrm{p}=0.044$; mientras que la tableta como dispositivo en todas sus comparaciones no revelan resultados notables en las pruebas post hoc aplicadas para esta herramienta Web 2.0.

La comparativa atendiendo a la valoración cercana a positiva de la wiki $\mathrm{F}(2,186)=4.585$ y $\mathrm{p}=0.011$ se proporciona entre el móvil y el PC, 
siendo el primer dispositivo más favorable $\mathrm{t}(186)=2.946$ y $\mathrm{p}=0.011$; en contra el dispositivo Tableta no refleja relevancia entre las comparaciones como indica las pruebas de Scheffé y Bonferroni.

El resto de herramientas Web 2.0, redes sociales y educativas, editores y buscadores de imágenes, buscadores de vídeo, buscadores de presentaciones y foros; no revelan diferencias significativas en la valoración de estas para la intervención en el ámbito de educación para la convivencia y la resolución de conflictos en relación al dispositivo que emplea la muestra para su acceso a Internet, en otros términos, la Anova no refleja resultados relevantes.

\subsection{Valoración herramientas Web 2.0 en función del acceso semanal de conexión}

El alumnado que se conecta a Internet todos los días realiza una mayor valoración de los entornos de aprendizaje virtuales para la intervención en el ámbito de la educación para la convivencia y resolución de conflictos frente a los que acceden 20 3 días $\mathrm{t}(186)=4.695$ y $\mathrm{p}=0.000$, y a los que lo hacen 4 o $5 \mathrm{t}(186)=4.799$ y $\mathrm{p}=0.000$; por el contrario la comparación entre el acceso 2 o 3 días y 4 o 5 no arrojan resultados notables en las pruebas post hoc aplicadas.

La muestra participante que accede todos los días a Internet valora casi positivamente los blog en comparación con los que lo hacen 2 o 3 días $\mathrm{t}(186)=4.958$ y $\mathrm{p}=0.000$ y, con los de 4 o 5 $\mathrm{t}(186)=4.874$ y $\mathrm{p}=0.000$ en el ámbito para la intervención en la educación para la convivencia y resolución de conflictos; mientras que las restantes comparaciones no son significativas.

La valoración cercana a positiva de las redes sociales y educativas se asigna a los estudiantes que se conectan todos los días a Internet contra los que lo hacen 2 o 3 días $\mathrm{t}(186)=5.375$ y $\mathrm{p}=0.000$ y con los que acceden 4 o 5 días $\mathrm{t}(186)=3.205$ y p $=0.005$; mientras que, la comparativa entre 4 o 5 y 2 o 3 días de conexión a Internet revela que los primeros sostienen una mejor valoración que los segundos $\mathrm{t}(186)=2.888$ y $\mathrm{p}=0.013$.

La comparativa al considerar la valoración de los editores de vídeo, que proporciona la conexión a Internet todos los días frente a 2 o 3 días a la semana, es favorable al primer tipo de acceso siendo el valor del mismo cercano a positivo, $\mathrm{t}(186)=5.959$ y $\mathrm{p}=0.000$ y, el contraste todos los días y 4 o 5 días a la semana, propicia a la primera $\mathrm{t}(186)=6.436 \mathrm{y}$ $\mathrm{p}=0.000$.
Los estudiantes que acceden todos los días a Internet valoran casi positivamente los editores de imágenes para el ámbito en la intervención en la educación para la convivencia y resolución de conflictos frente a los que se conectan 2 o 3 días $\mathrm{t}(186)=5.528$ y $\mathrm{p}=0.000$; de la misma forma, los que disfrutan de una conectividad diaria frente a los de 4 o 5 días presentan una mayor valoración para esta herramienta Web 2.0, los primeros respecto a los segundos, $\mathrm{t}(186)=4.812$ y $\mathrm{p}=0.000$; mientras que la comparación 4 o 5 días de conexión frente a 2 o 3 no revelan resultados notables en las pruebas post hoc aplicadas.

La valoración de los buscadores de imágenes para este ámbito es mejor en los participantes que se conectan todos los días a Internet en comparación con los que lo hacen 2 o 3 días $\mathrm{t}(186)=4.810$ y $\mathrm{p}=0.000$, igualmente el contrate con el acceso a Internet de 4 o 5 días $\mathrm{t}(186)=3.682$ y $\mathrm{p}=0.001$.

$\mathrm{El}$ alumnado, que disfruta de una conectividad a Internet todos los días, valora positivamente los buscadores de texto en relación a los de 2 o 3 días $\mathrm{t}(186)=6.096$ y $\mathrm{p}=0.000 ; \mathrm{y}$ a los que gozan de una conexión de 4 o 5 días a la semana t(186)=5.729 y $\mathrm{p}=0.001$; el contraste entre el acceso de 4 o 5 días y 2 o 3 días no revela resultados notables en las pruebas de Bonferroni.

Los estudiantes que acceden a Internet todos los días indican una valoración casi positiva de los buscadores de presentaciones para el ámbito de intervención de la educación para la convivencia y la resolución de conflictos frente a los que lo hacen 2 o 3 días $\mathrm{t}(186)=4.732$ y $\mathrm{p}=0.001$; y a los que acceden 4 o 5 días a la semana $\mathrm{t}(186)=3.259$ y $\mathrm{p}=0.004$.

El contraste atendiendo a la mejor valoración de la wiki se proporciona más en el alumnado que accede a Internet todos los días que a los que se conectan 2 o 3 días $\mathrm{t}(186)=5.036$ y $\mathrm{p}=0.000$; $\mathrm{y}$, a los de conectividad de 4 o 5 días $t(186)=5.558$ y $\mathrm{p}=0.001$.

Los estudiantes que gozan de una conexión diaria a Internet valoran mejor los foros para la intervención en el ámbito de educación para la convivencia y resolución de conflictos frente a los que lo hacen 2 o 3 días a la semana $t(186)=4.198$ y $\mathrm{p}=0.000$ y frente a los que acceden 4 o 5 días $\mathrm{t}(186)=3.944$ y $\mathrm{p}=0.000$; la comparación entre la conectividad de 4 o 5 y 2 o 3 días a la semana no proyecta resultados indicativos de significación.

Los buscadores de vídeo no revelan diferencias significativas en la valoración de estos para el ámbito de intervención de la educación para la convivencia y resolución de conflictos en relación al acceso semanal que efectúa la muestra. 
Tabla 2. Anova de la valoración del ámbito de intervención de la educación para la convivencia y resolución de conflictos de once herramientas Web 2.0 en función de la conexión semanal que realiza.

\begin{tabular}{|c|c|c|c|c|c|}
\hline & $\begin{array}{c}\text { Acceso por } \\
\text { semana }\end{array}$ & $\mathrm{N}$ & Media & $\mathrm{s}$ & F y p de los grupos favorables \\
\hline & 2 o 3 días & 25 & 2.74 & 1.228 & $\mathrm{~F}=17.607$ y $\mathrm{p}=0.000$ \\
\hline \multirow[t]{3}{*}{ Entornos de aprendizaje virtuales } & 4 o 5 días & 62 & 3.02 & 1.015 & favorable al acceso todos los \\
\hline & Todos los días & 101 & 3.80 & 1.018 & días \\
\hline & 2 o 3 días & 25 & 2.81 & 1.145 & $\mathrm{~F}=18.909$ y $\mathrm{p}=0.000$ \\
\hline \multirow[t]{3}{*}{$\mathrm{B} \log \mathrm{s}$} & 4 o 5 días & 62 & 3.12 & 1.000 & favorable al acceso todos los \\
\hline & Todos los días & 101 & 3.90 & 0.985 & días \\
\hline & 2 o 3 días & 25 & 2.74 & 1.347 & $\mathrm{~F}=16.066$ y $\mathrm{p}=0.000$ favorable \\
\hline \multirow[t]{3}{*}{ Redes sociales y educativas } & 4 o 5 días & 62 & 3.44 & 0.994 & al acceso todos los días y 4 o 5 \\
\hline & Todos los días & 101 & 3.97 & 1.018 & días \\
\hline & 2 o 3 días & 25 & 2.59 & 1.083 & $\mathrm{~F}=30.016$ y $\mathrm{p}=0.000$ \\
\hline \multirow[t]{3}{*}{ Editores de vídeo } & 4 o 5 días & 62 & 2.88 & 1.015 & favorable al acceso todos los \\
\hline & Todos los días & 101 & 3.92 & 1.025 & días \\
\hline & 2 o 3 días & 25 & 2.63 & 1.149 & $\mathrm{~F}=21.139$ y $\mathrm{p}=0.000$ \\
\hline \multirow[t]{3}{*}{ Editores de imágenes } & 4 o 5 días & 62 & 3.06 & 0.875 & favorable al acceso todos los \\
\hline & Todos los días & 101 & 3.80 & 0.999 & días \\
\hline & 2 o 3 días & 25 & 2.70 & 1.171 & $\mathrm{~F}=14.579$ y $\mathrm{p}=0.000$ \\
\hline \multirow[t]{3}{*}{ Buscadores de imágenes } & 4 o 5 días & 62 & 3.21 & 1.074 & favorable al acceso todos los \\
\hline & Todos los días & 101 & 3.85 & 1.102 & días \\
\hline & 2 o 3 días & 25 & 2.70 & 1.137 & \\
\hline \multirow[t]{3}{*}{ Buscadores de vídeo } & 4 o 5 días & 62 & 3.64 & 1.312 & $\mathrm{~F}=1.314$ y $\mathrm{p}=0.27$ \\
\hline & Todos los días & 101 & 3.77 & 1.026 & No sıgnifıcatıva \\
\hline & 2 o 3 días & 25 & 2.67 & 1.209 & $\mathrm{~F}=27.307$ y $\mathrm{p}=0.000$ \\
\hline \multirow[t]{3}{*}{ Buscadores de texto } & 4 o 5 días & 62 & 3.11 & 1.083 & favorable al acceso todos los \\
\hline & Todos los días & 101 & 4.06 & 1.003 & días \\
\hline & 2 o 3 días & 25 & 2.70 & 1.203 & $\mathrm{~F}=13.212$ y $\mathrm{p}=0.000$ \\
\hline \multirow[t]{3}{*}{ Buscadores de presentaciones } & 4 o 5 días & 62 & 3.24 & 0.978 & favorable al acceso todos los \\
\hline & Todos los días & 101 & 3.78 & 1.069 & días \\
\hline & 2 o 3 días & 25 & 2.78 & 1.086 & $\mathrm{~F}=22.020 \mathrm{y} \mathrm{p}=0.000$ \\
\hline \multirow[t]{3}{*}{ Wiki } & 4 o 5 días & 62 & 3.00 & 1.038 & favorable al acceso todos los \\
\hline & Todos los días & 101 & 3.91 & 1.028 & días \\
\hline & 2 o 3 días & 25 & 2.89 & 1.281 & $\mathrm{~F}=13.003$ y $\mathrm{p}=0.000$ \\
\hline \multirow[t]{2}{*}{ Foros } & 4 o 5 días & 62 & 3.18 & 0.943 & favorable al acceso todos los \\
\hline & Todos los días & 101 & 3.82 & 1.012 & días \\
\hline
\end{tabular}

\subsection{Valoración herramientas Web 2.0 en función del acceso diario de conexión}

Asimismo, replicando el Anova (n.s.=0.05), en esta ocasión atendiendo a la valoración en el ámbito de intervención de la educación para la convivencia y resolución de conflictos en función de las horas al días de conexión a Internet, los resultados más notables se muestran en la tabla 3 .

El alumnado, que se conecta a Internet más de 5 horas al día, valora positivamente los entornos de aprendizaje virtuales para la intervención en este ámbito frente a los que acceden 1 o 2 horas $\mathrm{t}(186)=5.279$ y $\mathrm{p}=0.000$, o la conectividad 3 o 4 horas $t(186)=2.740$ y $p=0.020$. Mientras que, los que acceden entre 3 o 4 horas al día indican una mejor valoración de esta herramienta frente a los que lo hacen 1 o 2 horas $\mathrm{t}(186)=3.118$ y $\mathrm{p}=0.006$.

La muestra participante, que accede más de 5 horas al día a Internet, indica una valoración positiva de los blog en comparación con los que lo hacen 1 o 2 horas al día $\mathrm{t}(186)=4.925$ y $\mathrm{p}=0.000$, y con los que se conectan entre 3 o 4 horas al día $t(186)=2.903$ y $\mathrm{p}=0.013$; igualmente, la comparación a través de la conectividad entre 3 o 4 frente a 1 o 2 horas al día arroja que a mayor número de horas de acceso mejor valoración $\mathrm{t}(186)=2.491$ y $\mathrm{p}=0.041$.

La valoración positiva en las redes sociales y educativas se asigna a los estudiantes que se conectan más de 5 horas al día frente a los que lo hacen 1 o 2 horas al día $\mathrm{t}(186)=4.673$ y $\mathrm{p}=0.000$, y con los que acceden entre 3 o 4 horas $t(186)=3.167$ y $\mathrm{p}=0.005$; por el contrario la comparación entre la conectividad 1 o 2 horas al día y entre 3 o 4 no 
arrojan resultados notables como señalan la prueba de Bonferroni aplicada.

Los estudiantes que acceden más de 5 horas al día a Internet valoran positivamente los editores de vídeo para la intervención en el ámbito frente a los que se conectan 1 o 2 horas al día $t(186)=5.540$ y $\mathrm{p}=0.000$. Igualmente, la comparación a través de la conectividad entre 3 o 4 horas frente a 1 o 2 al día arroja que a mayor horas de acceso mejor valoración de esta herramienta $\mathrm{t}(186)=3.862$ y $\mathrm{p}=0.000$.

La comparativa entre más de 5 horas y 1 o 2 horas al día de conexión a Internet es favorable a la primera atendiendo a una mejor valoración de los editores de imágenes $\mathrm{t}(186)=4.685$ y $\mathrm{p}=0.000 \mathrm{y}$, el contraste entre 3 o 4 horas al día, propicia, otra vez a la primera $\mathrm{t}(186)=2.974$ y $\mathrm{p}=0.010$; mientras que las restantes comparaciones no arrojan significatividad.

Una valoración positiva de los buscadores de imágenes en el ámbito de intervención de la educación para la convivencia y resolución de conflictos se promueve más en los participantes que se conectan más de 5 horas al día a Internet en comparación con los que lo hacen 1 o 2 al día $\mathrm{t}(186)=5.184$ y $\mathrm{p}=0.000 ; \mathrm{y}$, con los de entre 3 o 4 horas al día $\mathrm{t}(186)=2.885$ y $\mathrm{p}=0.013$. De la misma manera, los que acceden entre 3 o 4 horas al día indican una mejor valoración de esta herramienta frente a los que lo hacen 1 o 2 horas $\mathrm{t}(186)=2.786 \mathrm{y}$ $\mathrm{p}=0.017$.

El alumnado, que disfruta de un acceso a Internet de más de 5 horas, valora positivamente los buscadores de texto en relación a los de 1 o 2 horas al día $\mathrm{t}(186)=5.444$ y $\mathrm{p}=0.000$, y con los de entre $3 \mathrm{o}$ 4 horas $\mathrm{t}(186)=2.847$ y $\mathrm{p}=0.014$; asimismo, la comparación a través de la conectividad entre 3 o 4 horas frente a 1 o 2 horas al día arroja que a mayor horas de acceso mejor valoración de esta herramienta en el ámbito de intervención de la educación para la convivencia y resolución de conflictos $\mathrm{t}(186)=3.167$ y $\mathrm{p}=0.005$.

La valoración positiva de los buscadores de presentaciones se provoca en los estudiantes que acceden a Internet más de 5 horas al día frente a los que lo hacen 1 o 2 horas $\mathrm{t}(186)=5.098$ y $\mathrm{p}=0.000$; $\mathrm{y}$ a los que acceden entre 3 o 4 al día $t(186)=3.325$ y $\mathrm{p}=0.003$ ]; la conectividad de entre 3 o 4 horas frente a los de 1 o 2 al día no provoca significación.

El contraste atendiendo a la valoración de la herramienta wiki se proporciona entre el acceso a Internet de más de 5 horas y el de 1 o 2 al día, siendo la primera conexión más favorable, $\mathrm{t}(186)=4.898$ y $\mathrm{p}=0.000$. Igualmente, la conexión entre 3 o 4 al día provoca una mejor valoración frente al de 1 o 2 horas $\mathrm{t}(186)=3.462$ y $\mathrm{p}=0.002$.

Los estudiantes, que disfrutan de una conexión de más de 5 horas al día a Internet, señalan una valoración positiva de los foros en relación a los que lo hacen 1 o 2 horas $\mathrm{t}(186)=4.199$ y $\mathrm{p}=0.000$, y frente a los que acceden entre 3 o 4 horas al día $\mathrm{t}(186)=3.065$ y $\mathrm{p}=0.007$; por el contrario la comparación entre la conectividad 1 o 2 horas al día y entre 3 o 4 no arrojan resultados notables como señalan la prueba de Bonferroni aplicada.

Los buscadores de vídeo no revelan diferencias significativas en la valoración de esta herramienta Web 2.0 para el ámbito de intervención de la educación para la convivencia y resolución de conflictos en relación al acceso en horas al día que disfrutan los estudiantes.

Tabla 3. Anova de la valoración del ámbito de intervención de la educación para la convivencia y resolución de conflictos de once herramientas Web 2.0 en función de la conexión diaria que se realiza.

\begin{tabular}{|c|c|c|c|c|c|}
\hline \multirow{4}{*}{$\begin{array}{l}\text { Entornos de } \\
\text { aprendizaje } \\
\text { virtuales }\end{array}$} & Acceso por horas al día & $\mathrm{N}$ & Media & $\mathrm{S}$ & F y p de los grupos favorables \\
\hline & 1 o 2 horas & 70 & 2.95 & 1.019 & \multirow{3}{*}{$\begin{array}{c}\mathrm{F}=14.365 \text { y } \mathrm{p}=0.000 \text { favorable al acceso } \\
\text { de más de } 5 \text { horas y entre } 3 \text { o } 4 \text { horas }\end{array}$} \\
\hline & Entre 3 o 4 horas & 77 & 3.48 & 1.146 & \\
\hline & Más de 5 horas & 41 & 4.02 & 0.963 & \\
\hline \multirow{3}{*}{ Blogs } & 1 o 2 horas & 70 & 3.11 & 0.945 & \multirow{3}{*}{$\begin{array}{c}\mathrm{F}=12.191 \text { y } \mathrm{p}=0.000 \text { favorable al acceso } \\
\text { de más de } 5 \text { horas y entre } 3 \text { o } 4 \text { horas }\end{array}$} \\
\hline & Entre 3 o 4 horas & 77 & 3.52 & 1.178 & \\
\hline & Más de 5 horas & 41 & 4.09 & 0.921 & \\
\hline \multirow{3}{*}{$\begin{array}{l}\text { Redes sociales y } \\
\text { educativas }\end{array}$} & 1 o 2 horas & 70 & 3.28 & 1.117 & \multirow{3}{*}{$\begin{array}{c}\mathrm{F}=10.934 \text { y } \mathrm{p}=0.000 \text { favorable al acceso } \\
\text { de más de } 5 \text { horas }\end{array}$} \\
\hline & Entre 3 o 4 horas & 77 & 3.61 & 1.119 & \\
\hline & Más de 5 horas & 41 & 4.26 & 0.954 & \\
\hline \multirow{3}{*}{ Editores de vídeo } & 1 o 2 horas & 70 & 2.86 & 0.998 & \multirow{3}{*}{$\begin{array}{c}\mathrm{F}=16.647 \text { y } \mathrm{p}=0.000 \text { favorable al acceso } \\
\text { de más de } 5 \text { horas y entre } 3 \text { o } 4 \text { horas }\end{array}$} \\
\hline & Entre 3 o 4 horas & 77 & 3.54 & 1.219 & \\
\hline & Más de 5 horas & 41 & 4.02 & 0.963 & \\
\hline \multirow{3}{*}{$\begin{array}{l}\text { Editores de } \\
\text { imágenes }\end{array}$} & 1 o 2 horas & 70 & 3.05 & 0.890 & \multirow{3}{*}{$\begin{array}{c}\mathrm{F}=10.991 \text { y } \mathrm{p}=0.000 \text { favorable al acceso } \\
\text { de más de } 5 \text { horas }\end{array}$} \\
\hline & Entre 3 o 4 horas & 77 & 3.40 & 1.153 & \\
\hline & Más de 5 horas & 41 & 3.98 & 0.988 & \\
\hline Buscadores de & 1 o 2 horas & 70 & 3.04 & 1.066 & $\mathrm{~F}=13.556$ y $\mathrm{p}=0.000$ favorable al acceso \\
\hline
\end{tabular}




\begin{tabular}{cccccc}
\hline & & & & & \\
\hline imágenes & Entre 3 o 4 horas & 77 & 3.54 & 1.209 & de más de 5 horas y entre 3 o 4 horas \\
& Más de 5 horas & 41 & 4.14 & 0.966 & \\
Buscadores de & 1 o 2 horas & 70 & 3.54 & 1.356 & \\
vídeo & Entre 3 o 4 horas & 77 & 3.44 & 1.090 & F=0.367 y p=0.693 no significativa \\
& Más de 5 horas & 41 & 3.93 & 1.033 & \\
Buscadores de & 1 o 2 horas & 70 & 3.07 & 1.102 & \multirow{2}{*}{ F=15.236 y p=0.000 favorable al acceso } \\
texto & Entre 3 o 4 horas & 77 & 3.63 & 1.232 & de más de 5 horas y entre 3 o 4 horas \\
& Más de 5 horas & 41 & 4.23 & 0.868 & \\
Buscadores de & 1 o 2 horas & 70 & 3.08 & 1.017 & F=12.971 y p=0.000 favorable al acceso \\
presentaciones & Entre 3 o 4 horas & 77 & 3.45 & 1.146 & de más de 5 horas \\
& Más de 5 horas & 41 & 4.12 & 0.956 & \\
Wiki & 1 o 2 horas & 70 & 2.99 & 1.000 & \multirow{F}{*}{ F=13.019 y p=0.000 favorable al acceso } \\
& Entre 3 o 4 horas & 77 & 3.59 & 1.143 & de más de 5 horas \\
& Más de 5 horas & 41 & 4.00 & 1.091 & \\
Foros & 1 o 2 horas & 70 & 3.20 & 1.020 & F=8.902 y p=0.000 favorable al acceso \\
& Entre 3 o 4 horas & 77 & 3.44 & 1.090 & de más de 5 horas \\
\hline
\end{tabular}

\subsection{Valoración herramientas Web 2.0 en función del tipo de conexión}

Finalmente, al tratar de establecer diferencias estadísticamente significativas atendiendo al tipo de conexión o red empleada, aplicando para la misma una prueba $\mathrm{T}$ de Student (n.s.=0.05), los resultados más indicativos son los siguientes:

Las personas que utilizan un tipo de conexión pública indican una mayor valoración de las herramientas Web 2.0 entornos de aprendizaje virtuales $(\mathrm{t}=-2,131$ y $\mathrm{p}=0,034$, promedio $=3,60)$, blog $(\mathrm{t}=-2,190$ y $\mathrm{p}=0,030$, promedio $=3,69)$, redes sociales $y$ educativas $(\mathrm{t}=-2,064$ y $\mathrm{p}=0,040$, promedio $=3,82)$ y wiki $(\mathrm{t}=-2,544$ y $\mathrm{p}=0,012$, promedio $=3,69)$ para la intervención en el ámbito de la educación para la convivencia y resolución de conflictos que los estudiantes que emplean la red privada.

Contrariamente a estos resultados los editores y buscadores de vídeo, los editores y buscadores de imágenes, los buscadores de texto, los buscadores de presentaciones y los foros, herramientas Web 2.0 estudiadas en esta investigación, no arrojan datos significativos en la comparación de medias en función del tipo de conexión empleada considerando la valoración para el ámbito 1 que indican los participantes.

\section{DISCUSIÓN Y CONCLUSIONES}

El sistema educativo consciente de la necesidad de abordar el clima relacional que se genera en cualquier interrelación, ya sea formal e informal, ha regulado y mantenido el énfasis en la educación para la convivencia y la resolución de conflictos.
En este sentido, el educador social, como agente con competencias en el campo educativo, debe estar formado para dar respuesta a las dificultades o necesidades que surjan de las interacciones diarias escolares.

Estos elementos, convivencia y resolución de conflictos, se ven afectados por las nuevas formas de comunicación e interacción provenientes del empleo de las tecnologías, por lo que los diversos profesionales encargados del acto educativo deberían estimar su beneficio o perjuicio en las aulas, así como, la valoración que supone su empleo para acercar al estudiante a la realidad; este hecho, se lleva a cabo en distintas comunidades autónomas, como es el caso de Andalucía, que en su Informe de la convivencia escolar de 2016 señala como una de las actuaciones o buenas prácticas la figura del alumnado "ciberayudante" como referente para el uso de las tecnologías entre sus iguales.

En este sentido, los resultados de este estudio arrojan que los estudiantes del Grado de Educador Social de la Universidad Pablo de Olavide (Sevilla) valoran de forma más positiva las redes sociales y los buscadores de vídeo para intervenir en este ámbito, el primero de estos recursos tecnológicos tiene su origen en la comunicación y en las redes de interacción (Marín, Sampedro y Muñoz, 2015) por lo que es lógico que se señale como herramienta para la intervención educativa en el ámbito para la educación en la convivencia y resolución de conflictos. Los buscadores de vídeo, como indica López Hernáez y Sabater (2014, p. 146) “el aprendizaje de la violencia a través de la televisión y otros medios audiovisuales no siempre se produce por imitación de las conductas observadas" no tiene que ser visto por los agentes educativos como un recurso que incite al mal clima de convivencia, sino más bien, como una herramienta que puede ser 
empleada para la búsqueda de material educativo que propicie los valores democráticos; lo cual podemos apreciar en proyectos innovadores como el llevado a cabo en Cataluña, donde las actividades efectuadas para mejorar la convivencia en varios centros por los agentes sociales y educativos ha sido realizar un Cine Forum (Prieto, Lombarte y Montañez, 2013).

Otro de los resultados obtenidos en esta investigación, pone de relieve que el alumnado de los primeros cursos (primero y segundo) del Grado de Educador Social valora más positivamente las herramientas Web 2.0 abordadas para la intervención en el ámbito para la educación de la convivencia y resolución de conflictos, en concreto las redes sociales y educativas, los buscadores de imágenes y vídeos, y blogs, lo cual no es de extrañar dada la multitud de propuestas didácticas que favorecen el aprendizaje de la convivencia como conocimiento transversal educativo, tal y como indica Pariente y Perochena (2013).

Por otro lado, el empleo del móvil como dispositivo para la conexión condiciona la estimación que se posee de algunos recursos tecnológicos para la intervención en la convivencia, en este sentido estos estudiantes de Educación Social indican como más ventajosas para la intervención en el aprendizaje de la convivencia, las herramientas que están enfocadas a la colaboración (wiki, blogs y entornos de aprendizaje virtuales), a la publicación (editores de vídeo), a la interacción (entornos de aprendizaje virtuales) y a la información (buscadores de texto).

Finalmente, la frecuencia de acceso a Internet condiciona la estimación, tanto en días como en horas, los estudiantes del Grado de Educación Social de la Universidad Pablo Olavide (Sevilla) señalan que reconocen valiosas todas las herramientas, a excepción de los buscadores de vídeo, a mayor conectividad a Internet. Este resultado, contradice el grado de valoración que le atribuían a los buscadores de vídeo, consideramos que la explicación, al mismo, viene determinada por la investigación realizada por López Hernáez y Sabater (2014), que obtienen entre sus conclusiones que los medios audiovisuales repercuten en los comportamientos agresivos del alumnado de secundaria; posiblemente la idea de los futuros educadores sociales, que más frecuencia de empleo de Internet, implicará la posible existencia de peligro en este recurso electrónico al encontrar vídeos que no pertenezcan a las edades de los sujetos con los que se va a trabajar, en otros términos, que reflexionen sobre el beneficio para ellos mismos en su futura labor pero no como recurso didáctico tecnológico para sujetos menores de edad.

De una u otra forma, la convivencia y la resolución de conflictos deben abordarse desde una perspectiva asertiva, dado que estos elementos son clave para desarrollar principios democráticos como el respeto, la igualdad o la tolerancia, como indica Espuny et al. (2011) con las herramienta Web 2.0 redes sociales y educativas; sin embargo, no debemos olvidar que estas, en algunos casos pueden provocar formas de acoso como el cyberbullying (Ortega, Calmaestra y Mora, 2008); lo cual pone de manifiesto la relevancia de nuestro estudio, aunque los resultados no puedan ser generalizables, dado que el punto de vista del educador social como agente comunitario favorece la adopción de diversas estrategias educativas en la búsqueda de mejorar el clima en las aulas. Asimismo, la colaboración de este profesional, como su formación de forma concreta en los recursos tecnológicos, puede facilitar la implementación de proyectos y planes que propicie prácticas asertivas mediante un empleo adecuado de las herramientas Web 2.0 entre el alumnado y la comunidad escolar.

\section{FINANCIACIÓN}

Esta investigación ha sido financiada por la Consejería de Innovación de la Junta de Andalucía, a través del Grupo de investigación SEJO49 denominado Evaluación Educativa e Innovación.

\section{REFERENCIAS}

Bisquerra, R. (2008). Educación para la ciudadanía y convivencia. El enfoque de la Educación Emocional. Madrid, España: Wolters Kluwer.

Escorcia-Oyola, L. y Jaimes de Triviño, C. (2015). Tendencias de uso de las TIC en el contexto escolar a partir de las experiencias de los docentes. Educación y Educadores, 18(1), 137-152. DOI: 10.5294/edu.2015.18.1.8

Espuny, C.; González, J.; Lleixà, M. y Gisbert, M. (2011). Actitudes y expectativas del uso educativo de las redes sociales en los alumnos universitarios. RUSC. Revista de Universidad y Sociedad del Conocimiento, 8(1). $171-185$.

Fullana, J.; Pallisera, M. y Planas, A. (2011). Las competencias profesionales de los educadores sociales como punto de partida para el diseño curricular de la formación universitaria. Un estudio mediante el método Delphi. Revista Iberoamericana de Educación, 56(1), 1 - 13.

González, M., Olmos, S. y Serrate, S. (2015). Pensamiento y acción socioeducativa en contextos de enseñanza secundaria. Un estudio descriptivo-correlacional. Teoría de la Educación. Revista Interuniversitaria, 27(2), 91-114. DOI: http://dx.doi.org/10.14201/teoredu201527291114 
Hernández, R., Fernández, C. y Baptista, P. (2014). Metodología de la investigación $\left(6^{\mathrm{a}}\right.$. ed.). México: McGraw Hill Interamericana.

López Hernáez, L. y Savater, C. (2014). Medios audiovisuales y acoso escolar: buenas prácticas para la prevención y promoción de la convivencia. Revista de Investigación en Educación, 12(2), 145 - 163.

Marín, V., Sampedro, B.E. y Muñoz, J.M. (2015). ¿Son adictos a las redes sociales los estudiantes universitarios? Revista Complutense de Educación, 26, 233-251. DOI: http://dx.doi.org/10.5209/rev_RCED.2015.v26.46659

Meza, L.G. (2002). Metodología de la investigación educativa: posibilidades de integración. Revista Comunicación, 12 (1) (23), 182 - 194. Recuperado de: http://www.tec-

digital.itcr.ac.cr/servicios/ojs/index.php/comunicacion/ article/view/1223/1128.

Morales, P. (2007). La fiabilidad de los test y las escalas. Madrid: Universidad Pontificia Comillas. Recuperado de

http://web.upcomillas.es/personal/peter/estadisticabasi ca/Fiabilidad.pdf.

Ortega, R.; Calmaestra, J. y Mora, J. (2008). Cyberbullying. International Journal of Psychology and Psychological Therapy, 8(2), $183-192$.

Pariente, J. L. y Perochena, P. (2013). Didáctica de la educación en valores en la ESO. Una propuesta utilizando las tecnologías para el aprendizaje y el conocimiento. Pixel Bit. Revista de Medios y Educación, 42, 195 208.

Prieto, R.; Lombarte, S. y Montañez, X. (2013). Desmontemos rumores para fomentar la convivencia. Educación Social. Revista de Intervención Socioeducativa, 54, $123-141$

Rodríguez Izquierdo, R. M. (2011). Estudio sobre el proceso de integración laboral de las educadoras y educadores sociales en Andalucía. REOP. Revista Española de Orientación y Psicopedagogía, 22(3), 314 - 328. DOI: http://dx.doi.org/10.5944/reop.vol.22.num.3.201 1.11284

Sabariego, M. (2012). La investigación educativa: génesis, evolución y características. En Bisquerra, R. (coord.). Metodología de la investigación educativa. (pp. 51 87). ( $3^{\text {a }}$. ed.). Madrid, España: La Muralla. 